\title{
High-repetition-rate and high-power picosecond regenerative amplifier based on a single bulk Nd:GdVO 4 crystal
}

\author{
Jie Guo ${ }^{1}$, Wei Wang ${ }^{1,2}$, Hua Lin ${ }^{1}$, and Xiaoyan Liang ${ }^{1}$ \\ ${ }^{1}$ State Key Laboratory of High Field Laser Physics, Shanghai Institute of Optics and Fine Mechanics, Chinese Academy of Sciences, \\ Shanghai 201800, China \\ ${ }^{2}$ Center of Materials Science and Optoelectronics Engineering, University of Chinese Academy of Sciences, Beijing 100049, China \\ (Received 19 January 2019; revised 21 March 2019; accepted 4 April 2019)
}

\begin{abstract}
We report on a high-repetition-rate, high-power continuously pumped $\mathrm{Nd}: \mathrm{GdVO}_{4}$ regenerative amplifier. Numerical simulations successfully pinpoint the optimum working point free of bifurcation instability with simultaneous efficient energy extraction. At a repetition rate of $100 \mathrm{kHz}$, a maximum output power of $23 \mathrm{~W}$ was obtained with a pulse duration of $27 \mathrm{ps}$, corresponding to a pulse energy of $230 \mu \mathrm{J}$. The system displayed an outstanding stability with a root mean square power noise as low as $0.3 \%$. The geometry of the optical resonator and the pumping scheme enhanced output power in the $\mathrm{TEM}_{00}$ mode with a single bulk crystal. Accordingly, nearly diffraction-limited beam quality was produced with $M^{2} \approx 1.2$ at full pump power.
\end{abstract}

Keywords: diode-pumped solid state laser; high repetition rate regenerative amplifier; picosecond laser

\section{Introduction}

Compact, stable and high-power diode-pumped shortpulse laser amplifier systems with excellent spatial quality are ideal sources for high-power optical parametric chirped pulse amplification (OPCPA) and efficient laser processing $^{[1-5]}$. Among various configurations, regenerative amplifiers (RAs) are routinely used to enhance the output of mode-locked oscillators because of their ability to provide gains of several orders of magnitude and a resonator structure that maintains the spatial quality of the seed ${ }^{[6-9]}$.

In the past two decades, various solutions and architectures have been proposed for power scaling of regenerative amplifiers. The thin-disk geometry was particularly impressive for heat dissipation. Nubbemeyer et al. employed a Kerrlens mode-locked thin-disk oscillator to seed two stages of regenerative amplifiers, including a thin-disk preamplifier and a main amplifier comprising two thin-disk modules, generating $>200 \mathrm{~mJ}$ pulses at a $5 \mathrm{kHz}$ repetition rate and $>100 \mathrm{~mJ}$ at a $10 \mathrm{kHz}$ repetition rate ${ }^{[10]}$. The cryogenic cooling technique offered another alternative to obtain a high pulse energy at a $1 \mathrm{kHz}$ repetition rate, although measures were necessary to mitigate the reduced gain bandwidth ${ }^{[11]}$.

Correspondence to: X. Liang, No. 390 Qinghe Road, Jiading, Shanghai 201800, China. Email: liangxy@siom.ac.cn
Although not so aggressive in heat removal, laser systems based on bulk materials generally employ simpler structures and work more efficiently. These characteristics make bulk materials favored choices as laser media in high-power diode-pumped solid state lasers ${ }^{[12-14]}$. An average output power of $34 \mathrm{~W}$ with pulses as short as $140 \mathrm{fs}$ at a $500 \mathrm{kHz}$ repetition rate has been achieved by a bulk Yb:CALGO regenerative amplifier with the implementation of the chirped pulse amplification (CPA) technique ${ }^{[15]}$.

High-repetition-rate regenerative amplifiers are beneficial for promoting industrial throughput and reducing data accumulation or processing time in scientific applications. However, high repetition rates come with bifurcation and even chaotic pulse train dynamics, in which the pulse energy is unstable ${ }^{[16]}$. This bifurcation-termed phenomenon constitutes a major impediment for RAs operated at high repetition rates ${ }^{[3]}$, where the operating parameters of the laser systems have to be carefully optimized to maintain equilibrium $^{[17-20]}$. A detailed analysis concerning this problem is elaborated in the following section. For more than a decade, several methods have been proposed to eliminate this deleterious effect. The methodologies include raising the seed energy ${ }^{[19,21]}$, pumping at higher intensity ${ }^{[17]}$, operating at the second operating point outside the unstable region if the effect could not be thoroughly avoided ${ }^{[22]}$, 
or working in a bistable regime with a negligible lower bifurcation branch ${ }^{[23]}$. Consequently, efforts to optimize system parameters are necessary to achieve stable and efficient performance.

RAs based on bulk neodymium gain media show specific advantages. A high stimulated-emission cross-section simplifies the system design, and requirements such as the reflectivity for optical components are allayed. RAs based on them are generally free of CPA because of the relatively longer pulse durations. Diode-pumped RAs built with Nd:YAG or Nd:YLF crystals are commonly side-pumped for high pulse energy generation ${ }^{[24-29]}$. End-pumping schemes provide simpler structures and are always realized by neodymium-doped vanadate crystals. Lasers based on neodymium-doped vanadate crystals deliver moderate pulse energies as well as average power, but less complex cavities and pumping schemes are required. In 2013, a regenerative amplifier based on a $\mathrm{Nd}: \mathrm{YVO}_{4}$ crystal delivering a $28 \mathrm{~mJ}$ pulse energy at a $1 \mathrm{kHz}$ repetition rate and a $112.1 \mathrm{ps}$ long pulse was reported ${ }^{[30]}$. The whole setup was rather complex because a CPA configuration had to be used and the multipulse output limited its applications. Seeded by a cavitydumped laser, a $10.5 \mathrm{~W}$ output power was generated with a Nd: $\mathrm{YVO}_{4} \mathrm{RA}$ by the same group in 2015, although the repetition rate was just $10 \mathrm{kHz}^{[31]}$. For high repetition rate reports, an average output power of $25.5 \mathrm{~W}$ at $200 \mathrm{kHz}$ was realized in 2008 by Coherent Inc. ${ }^{[32]}$. In 2012, a continuous-wave $(\mathrm{CW})$ beam of a distributed feedback (DFB) diode laser modulated by an ultra-fast semiconductor modulator was injected into a regenerative amplifier based on a Nd: $\mathrm{YVO}_{4}$ crystal to produce $47.7 \mathrm{~W}$ at $816 \mathrm{kHz}$ and $26.4 \mathrm{~W}$ at $99.8 \mathrm{kHz}$, with pulse durations adjustable from $400 \mathrm{ps}$ to $1 \mathrm{~ns}^{[33]}$. However, this scheme suffered from amplitude fluctuations and $\mathrm{CW}$ background noise, which are disadvantageous for many applications. Among vanadate crystals, $\mathrm{Nd}: \mathrm{LuVO}_{4}$ has the highest stimulated emission cross-section and was applied to achieve $25.1 \mathrm{~W}$ at $1.43 \mathrm{MHz}$ and $20.5 \mathrm{~W}$ at $100 \mathrm{kHz}$ by our group in $2016^{[21]}$. However, beam quality degradation in long-term operation was observed in this $\mathrm{Nd}: \mathrm{LuVO}_{4}$ dual-crystal configuration RA, which may be attributed to the immature manufacturing technology of this commercially unavailable gain medium. In Refs. [14, 34], Nd: $\mathrm{YVO}_{4}$ crystals as long as $20 \mathrm{~mm}$ and $30 \mathrm{~mm}$ were used, respectively, to produce relatively low extraction efficiencies. The authors experimentally observed the bifurcation phenomena for their specific working conditions by adjusting the number of cavity round trips (NRTs) in the RA resonators; however, no detailed study on the physical process was carried out. As a result, numerous trials had to be implemented in practice until the output pulse became stable.

Here we report a $100 \mathrm{kHz}$ high-power regenerative amplifier based on a single bulk Nd:GdVO 4 crystal. The basic properties of three Nd-doped single crystals are compared
Table 1. Basic properties of three Nd-doped single crystals (see Refs. [35-42]).

\begin{tabular}{|c|c|c|c|}
\hline Parameter & Nd:YAG & $\mathrm{Nd}: \mathrm{YVO}_{4}$ & $\mathrm{Nd}: \mathrm{GdVO}_{4}$ \\
\hline Pump wavelength (nm) & 808869885 & 808880888 & 808880888 \\
\hline Emission wavelength (nm) & 1064 & 1064 & 1063 \\
\hline Emission bandwidth (nm) & 0.45 & 0.8 & 1.25 \\
\hline $\begin{array}{l}\text { Efficient emission cross-section } \\
\text { at } 1064 \mathrm{~nm}\left(10^{-19} \mathrm{~cm}^{2}\right)\end{array}$ & 2.8 & $\begin{array}{c}13.5(\pi) \\
6.5(\sigma)\end{array}$ & $\begin{array}{l}7.6(\pi) \\
1.2(\sigma)\end{array}$ \\
\hline Upper state lifetime $(\mu s)$ & 230 & 91 & 90 \\
\hline $\begin{array}{l}\text { Nonlinear refractive index } \\
\qquad\left(10^{-16} \mathrm{~cm}^{2} / \mathrm{W}\right)\end{array}$ & 8.1 & 14.7 & 12.6 \\
\hline $\begin{array}{l}\text { Thermal conductivity at } 300 \mathrm{~K} \\
(\mathrm{~W} / \mathrm{mK})\end{array}$ & 14 & $\begin{array}{l}5.1(\mathrm{a}) \\
5.23(\mathrm{c})\end{array}$ & $\begin{array}{l}10.1 \text { (a) } \\
11.4(\mathrm{c})\end{array}$ \\
\hline $\begin{array}{l}\text { Absorption cross-section at } \\
808 \mathrm{~nm}\left(10^{-19} \mathrm{~cm}^{2}\right)\end{array}$ & 0.41 & $\begin{array}{l}2.7(\pi) \\
1.2(\sigma)\end{array}$ & $\begin{array}{l}5.2(\pi) \\
1.23(\sigma)\end{array}$ \\
\hline Refractive index at $1064 \mathrm{~nm}$ & 1.820 & $\begin{array}{l}2.165(\mathrm{e}) \\
1.957(\mathrm{o})\end{array}$ & $\begin{array}{l}2.192(\mathrm{e}) \\
1.972(\mathrm{o})\end{array}$ \\
\hline
\end{tabular}

in Table ${ }^{[35-42]}$. Among these, $\mathrm{Nd}: \mathrm{GdVO}_{4}$ is an attractive candidate for high-power lasers because of its considerably higher thermal conductivity, which endows it with an ideal combination of the merits of $\mathrm{Nd}: \mathrm{YVO}_{4}$ and $\mathrm{Nd}: \mathrm{YAG}^{[35,43]}$. Numerical simulations were conducted to analyze the system stability and efficiency performance of the continuously pumped high-repetition-rate regenerative amplifier. In virtue of the analysis, optimized operating conditions were proposed and further realized in our experiment with a highly stable and efficient output. An average output power of $23 \mathrm{~W}$ at a repetition rate of $100 \mathrm{kHz}$ was delivered with an excellent output beam quality, characterized by $M^{2} \approx 1.2$. To the best of our knowledge, this is the highest output power that has been generated at $\sim 100 \mathrm{kHz}$ repetition rates for bulk crystal end-pumped all-solid-state picosecond regenerative amplifiers with pulse durations below $30 \mathrm{ps}$.

\section{Numerical simulations}

Looking at its working principle, one RA operation cycle can be divided into two successive stages: the pump stage and the amplification stage. No voltage is applied to the Pockels cell during the pump stage. The amplification stage starts when the seed is injected into the amplification cavity and a high voltage is applied to the Pockels cell. When the pulse energy reaches a certain level, the high voltage is switched off, the amplified pulse is ejected and the next cycle begins ${ }^{[20]}$. For continuously pumped regenerative amplifiers, this operation cycle division is justified when the amplification stage duration is considerably shorter than the inverse of the repetition rate so that the impact of the pump during amplification can be neglected. Nevertheless, for 
continuously pumped regenerative amplifiers, the operation cycles become interdependent when the pump stage duration becomes comparable to or even shorter than the upper state lifetime of the respective transition. Let us consider a $100 \mathrm{kHz}$ RA based on a Nd:GdVO 4 crystal as an example. The pump stage duration $(<10 \mu \mathrm{s})$ is shorter than the upper state lifetime of the ${ }^{4} \mathrm{~F}_{3 / 2}{ }^{4} \mathrm{I}_{11 / 2}$ transition $(90 \mu \mathrm{s})$. Then the population inversion accumulated during one operation cycle cannot be fully dissipated by spontaneous emission and nonradiative processes to reset the inversion state before the next pulse arrives. As a result, the output pulse energy depends on the previous energy extraction condition. In this situation, a stable output pulse energy can still be obtained by an appropriate choice of system parameters. A preliminary picture including crucial system parameters will be helpful to achieve a stable and efficient operating performance.

Numerical simulations were performed to fully exploit the system's capability: to ensure stable operation and extract as much stored energy as possible. Diagrams presented in the parameter space are very helpful to realize this prospect ${ }^{[19,20,44]}$. The parameter space separatrix and curve of NRT ${ }^{\mathrm{MAX}}$ are depicted in the same diagram. The separatrix divides the parameter space into two sections: corresponding to the stable and the bifurcation regimes. As described in Refs. [19, 20, 44], the separatrix shrinks to an ellipse for higher seed energy. NRT ${ }^{\mathrm{MAX}}$ presents the number of cavity round trips, potentially providing the highest output energy ${ }^{[19,44]}$. Optimum operation was reached when the curve of $\mathrm{NRT}^{\mathrm{MAX}}$ was outside the bifurcation region. The aforementioned parameter space includes the repetition rate and NRT. Other fundamental factors influencing the operating regimes involve the seed energy, pumping intensity and intracavity loss. Our simulations are established on the model developed by Grishin et al. ${ }^{[44]}$, which is based on one-dimensional discrete-time dynamical systems ${ }^{[45]}$. This model is found to have a fast computation speed and is adequate for our picosecond system with a narrow spectrum bandwidth. The parameters used in our simulations are listed in Table 2. These parameters are consistent with the conditions we planned to utilize in our experiment.

The basic equations describing the pump and amplification stages are listed as Equations (1) and (2), which were rearranged from rate equations ${ }^{[20]}$. For the pump stage, the initial and final gains were connected by the analytical solution to this set of equations, because no lasing was assumed. The cavity loss was included in the amplification stage, and solutions to Equations (1) and (2) were calculated numerically. The definitions and expressions for nondimensional terms are listed after the equations ${ }^{[20,44]}$.

$$
\begin{aligned}
\frac{\mathrm{d} \varepsilon(\tau)}{\mathrm{d} \tau} & =\varepsilon(\tau)\left[g(\tau)-g_{t}\right], \\
\frac{\mathrm{d} g(\tau)}{\mathrm{d} \tau} & =-\varepsilon(\tau) g(\tau)+\frac{1-g(\tau)}{\tau_{1}}, \\
G_{0} & =R_{p} T_{1} \sigma L_{a},
\end{aligned}
$$

Table 2. Key parameter values for simulation.

\begin{tabular}{lccc}
\hline Parameter & Value & Parameter & Value \\
\hline $\begin{array}{l}\text { Stable state small signal } \\
\text { gain } G_{0}\end{array}$ & 1.91 & Round trip loss $l$ & 0.04 \\
$\begin{array}{l}\text { Emission cross-section } \\
\sigma\left(\mathrm{cm}^{2}\right)\end{array}$ & $7.6 \times 10^{-19}$ & $\begin{array}{c}\text { Upper state lifetime } \\
T_{1}(\mu \mathrm{s})\end{array}$ & 90 \\
\hline
\end{tabular}

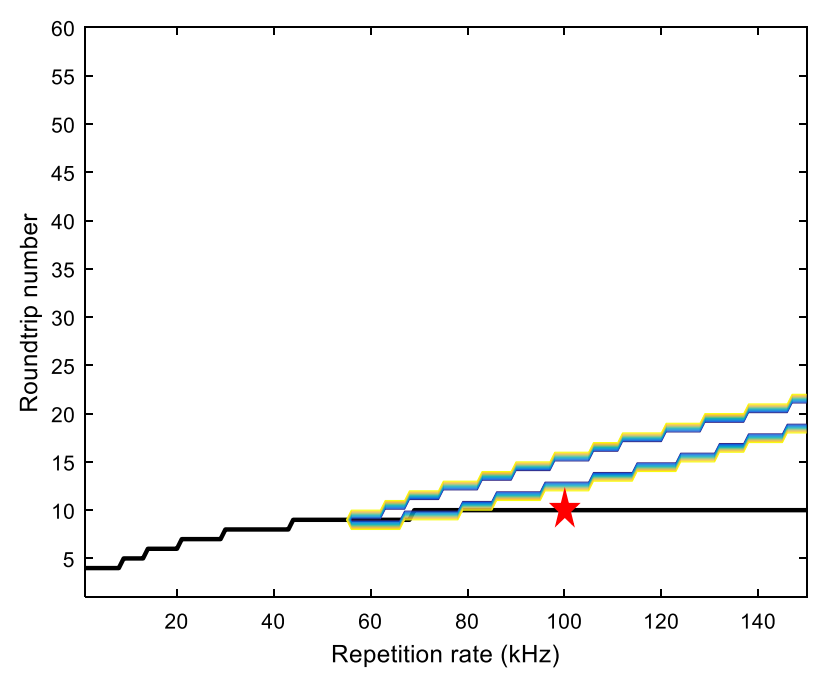

Figure 1. Parameter separatrix (colored blue and yellow) and curve of NRT $^{\text {MAX }}$ (colored bold black). The red star indicates the optimum working point for $100 \mathrm{kHz}$ repetition rate operation.

$$
\begin{aligned}
g & =N \sigma L_{a} / G_{0}, \\
\varepsilon & =\varphi \sigma /\left(A_{a} G_{0}\right), \\
\tau & =t G_{0} \beta / T_{0}, \\
g_{t} & =-\ln (1-l) / G_{0} .
\end{aligned}
$$

Here $R_{p}$ is the pumping rate, $L_{a}$ is the gain medium length, $N$ is the population inversion density, $\varphi$ is the photon number, $A_{a}$ is the mode cross-section in the gain medium, $\beta$ is the number of passes through the gain medium during one round trip ( $\beta=2$ for linear cavity; $\beta=1$ for ring cavity), $T_{0}$ is the cavity round trip time, $t$ is the time, $\tau_{1}$ is the normalized upper state lifetime $\left(\tau_{1}=T_{1} G_{0} \beta / T_{0}\right), \varepsilon$ is the normalized energy, and $\tau$ is the normalized round trip number. The definitions of other parameters can be found in Table 2.

In the simulation, the repetition rate of the RA operation range was set between $1 \mathrm{kHz}$ and $150 \mathrm{kHz}$. For the seed energy, the mode mismatch between the seed and the RA in the spatial and spectral domains should be taken into account ${ }^{[19]}$. The effective seed energy was estimated to be $\sim 40 \%$ of the seed energy available (800 $\mathrm{nJ}$ ). The simulation results are illustrated in Figure 1. The lower-right ellipticallike separatrix splits the stable and bifurcation regions, the inner of which is the bifurcation region. That is, if a combination of system parameters (repetition rate and round 
trip number) is chosen outside of the region surrounded by the elliptical-like separatrix then stable operation would be expected. The separatrix has a jagged appearance because in reality the NRT should be integers; this is also the reason for the form of the bold black curve of NRT ${ }^{\mathrm{MAX}}$. Diagrams of this type help us to estimate the optimum NRT when a specific repetition rate is required to exclude ranges inappropriate for operation. It can be seen from the diagram that outside a repetition rate range of $56-78 \mathrm{kHz}$, optimum operation could be achieved in theory. Consequently, with the seed source available, efficient and stable operation at a $100 \mathrm{kHz}$ repetition rate is attainable at a predicted round trip number of 10 , which is indicated with the red star.

\section{Experimental setup}

The experimental setup of the regenerative amplifier system is shown in Figure 2. The front-end seed source was based on a robust home-built, continuous wave passively modelocked picosecond $\mathrm{Nd}: \mathrm{GdVO}_{4}$ oscillator delivering an output power of $7.6 \mathrm{~W}$ at a $7.7 \mathrm{MHz}$ repetition rate. The oscillator avoided the complexity of a preamplifier and delivered excellent beam quality and long-term stability, ensuring reliable and flexible seeding of the amplifier with ample energy. An optical isolator was also included to protect the oscillator against potential feedback from the amplifier. Then a $\mathrm{RbTiOPO}_{4}(\mathrm{RTP})$ pulse picker reduced the repetition rate to $100 \mathrm{kHz}$ to enhance pulse contrast and suppress parasitic consumption of the stored energy. In addition, the lens between M2 and M3 matches the beam divergence and waist location between the modes of the oscillator and RA.

For the amplifier, the gain medium was a 0.5 at.\% doped $\mathrm{Nd}: \mathrm{GdVO}_{4}$ crystal with dimensions $4 \mathrm{~mm}(a) \times 4 \mathrm{~mm}$ $(c) \times 15 \mathrm{~mm}(a)$. Each polished facet of the crystal was antireflection coated at $879 \mathrm{~nm}$ and $1063 \mathrm{~nm}$. The crystal was wrapped with indium foil and mounted in a watercooled copper heatsink. The cooling water temperature was maintained at $15^{\circ} \mathrm{C}$ during operation. A linear polarized pump was imaged onto the $\mathrm{Nd}: \mathrm{GdVO}_{4}$ crystal of diameter $1100 \mu \mathrm{m}$ by a relay imaging system, in which a $\sigma$ polarized pumping configuration was adopted ${ }^{[46]}$. The pumping wavelength was centered at $879 \mathrm{~nm}$. Approximately $85 \%$ of the pump power was absorbed during a single pass through the crystal.

The RA cavity comprised a dichroic mirror M7, a quarterwave plate (QWP), a thin-film polarizer (TFP), a Pockels cell (PC) based on BBO crystal with an aperture of $5 \mathrm{~mm}$, and two highly reflective concave mirrors M5 $(R=600 \mathrm{~mm})$ and M6 $(R=300 \mathrm{~mm})$ along with two plane mirrors. Concave mirrors M5 and M6 were applied to get an appropriate beam size in the PC. The total cavity length of $1.96 \mathrm{~m}$ corresponds to a round trip time of about $13.1 \mathrm{~ns}$, which was adapted to the rise and fall times (about $10 \mathrm{~ns}$ ) of the PC driver. Although the high-repetition-rate RA benefits productivity in

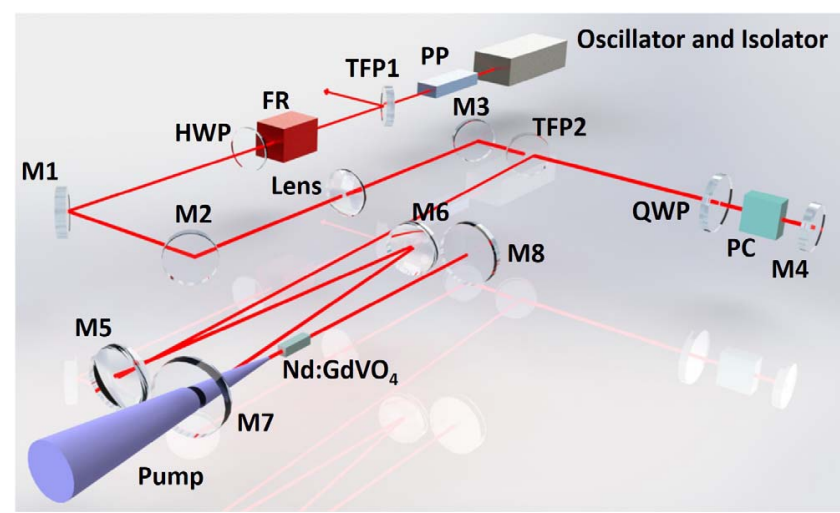

Figure 2. Schematic of the experimental setup: PP, pulse picker; TFP, thinfilm polarizer; FR, Faraday rotator; HWP, half-wave plate; QWP, quarterwave plate; PC, Pockels cell. The beam inside the RA cavity propagates along the $15 \mathrm{~mm}$ long $a$-axis of the $\mathrm{Nd}: \mathrm{GdVO}_{4}$ crystal.

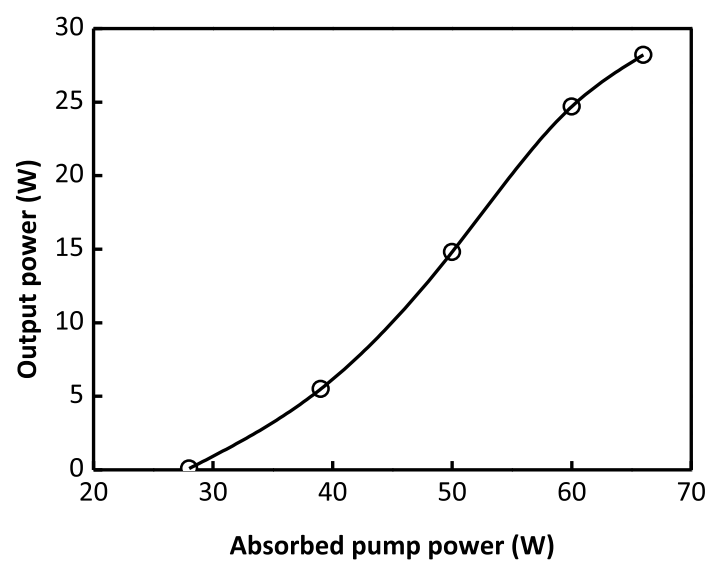

Figure 3. CW output power versus absorbed pump power.

industrial and scientific applications, it also poses challenges for the speed of high-voltage devices. A repetition rate of $100 \mathrm{kHz}$ was chosen for the investigation because it is a suitable compromise.

\section{Experimental results and discussions}

Continuous wave $(\mathrm{CW})$ operation was first conducted to confirm the optimum cavity performance, in which the combination of TFP2 and QWP served as the output coupler and the PC driver was switched off. Figure 3 shows the continuous-wave output power versus the absorbed pump power. A maximum output power of $28 \mathrm{~W}$ was achieved at $66 \mathrm{~W}$ absorbed pump power, which corresponds to $42 \%$ optical efficiency. The moderate efficiency was due to losses caused by the insertion of the PC, as the output power increased to $33 \mathrm{~W}$ once the $\mathrm{PC}$ was removed. The roll-over phenomenon in Figure 3 was attributed to thermal lensing of the $\mathrm{Nd}: \mathrm{GdVO}_{4}$ crystal, which destabilized the cavity. A similar phenomenon has been observed previously ${ }^{[47]}$. 

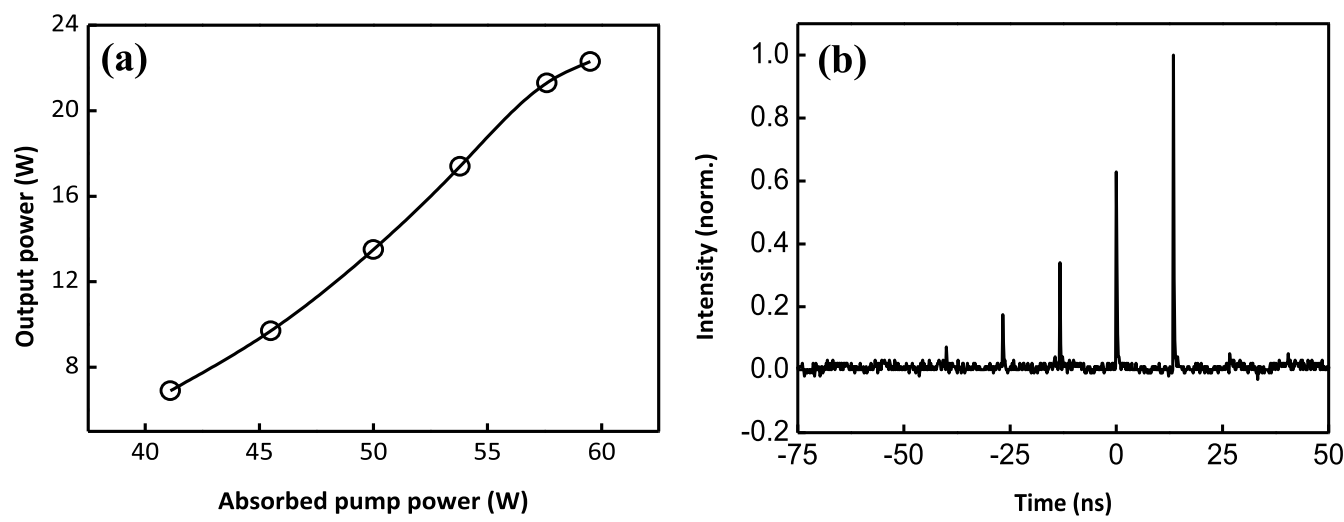

Figure 4. (a) RA regime output power versus absorbed pump power; (b) the last five intracavity signals of the RA.

In the RA operation regime, the seed with $800 \mathrm{~nJ}$ pulse energy was injected into the RA for amplification. Its output power is depicted in Figure 4(a). The onset of the parasitic cavity-dumped background was observed when the absorbed pump power was more than $60 \mathrm{~W}$. Therefore, the operation point was fixed around $60 \mathrm{~W}$ pumping power, which generated an average output power of $23 \mathrm{~W}$. A fast photodiode was used to monitor the intracavity signal through the leakage radiation behind $\mathrm{M} 4$, the evolution of which is shown in Figure 4(b) (only the last five signals could be observed in this way). The optimum round trip number was experimentally found to be 10 , which agreed well with the previous numerical simulations (indicated with the red star in Figure 1). System operation under altered conditions was also surveyed. When the round trip numbers were set as 9 and 11, the output power decreased by $300 \mathrm{~mW}$ and $500 \mathrm{~mW}$, respectively; however, pulse trains remained stable. Nevertheless, when the round trip number was set as 13 , the system started converting to the period-doubling regime. These performances confirmed the feasibility of our numerical simulations.

The temporal characteristics of the oscillator and RA output pulse were confirmed by an intensity autocorrelator. As shown in Figure 5, the pulse duration (FWHM) of the RA was determined to be $27 \mathrm{ps,} \mathrm{which} \mathrm{was} \mathrm{almost} \mathrm{the} \mathrm{same}$ as that of the seed. We also tried to measure the spectral properties of the seed and RA output separately. They were observed to be $<0.1 \mathrm{~nm}$ in width. Because of the limited resolution of our spectrometer $(\sim 0.04 \mathrm{~nm})$, accurate spectral widths and spectra of the oscillator and RA output are not provided here. Additionally, the calculated B-integral amounts to $2.1 \mathrm{rad}$, accounting for the polarizer, Pockels cell, and crystal. This value can be reduced to $1.9 \mathrm{rad}$ if the number of cavity round trips is reduced to 9 .

The long-term stability of the RA system at full pump power for $30 \mathrm{~min}$ is characterized and presented in Figure 6(a). The fluctuations of the average output power amount to $0.3 \%$ and the pulse-to-pulse stability was measured to be $1.7 \%$ RMS (root mean square), in which 1000

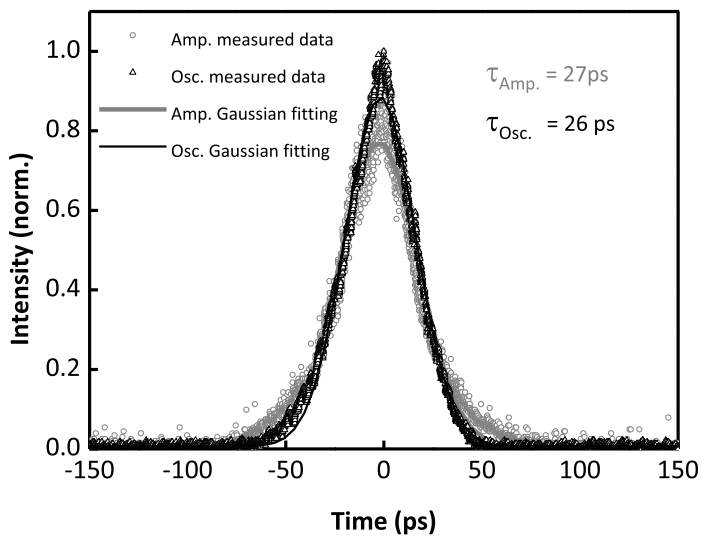

Figure 5. Intensity autocorrelation traces of the oscillator and RA output.

consecutive pulses were recorded within a time window of $10 \mathrm{~ms}$. The beam profile is demonstrated in Figure 6(b) with $M^{2} \approx 1.2$ at the maximum output power. The inset in Figure 6(b) displays the far-field beam profile at the focus.

\section{Conclusion}

In conclusion, we have demonstrated a $100 \mathrm{kHz}$ high-power continuously pumped $\mathrm{Nd}: \mathrm{GdVO}_{4}$ regenerative amplifier. The numerical analysis of the continuously pumped highrepetition-rate RA prior to the experiment facilitated optimization of the parameters in our experiment. This helped eliminate bifurcation instability and achieve efficient energy extraction. With a single bulk crystal, a maximum output pulse energy of $230 \mu \mathrm{J}$ at a repetition rate of $100 \mathrm{kHz}$ was obtained with a pulse duration of $27 \mathrm{ps}$ and root mean square power noise as low as $0.3 \%$. The $\sigma$ polarized pumping scheme and careful design of the optical resonator enhanced the output power in the $\mathrm{TEM}_{00}$ mode with a single bulk crystal. Accordingly, a nearly diffraction-limited beam quality was produced with $M^{2} \approx 1.2$ at full pump power. This is, to the best of our knowledge, the highest output 

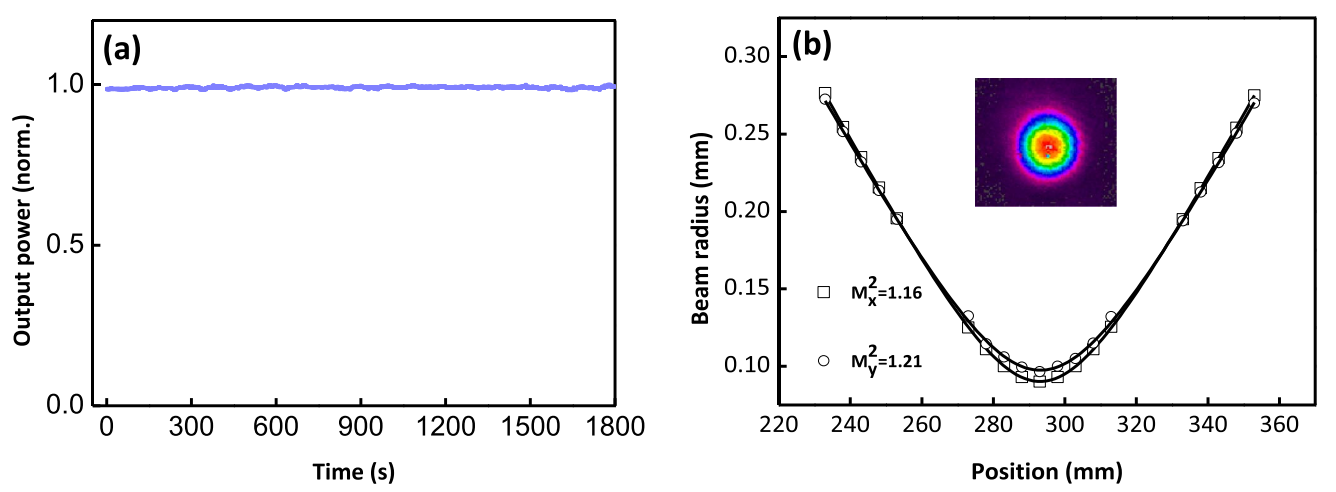

Figure 6. (a) Long-term power stability measurement of the RA output. (b) RA output beam quality.

power that has been attained at $\sim 100 \mathrm{kHz}$ repetition rates for a bulk crystal end-pumped all-solid-state picosecond regenerative amplifier with pulse durations below $30 \mathrm{ps}$. Power scaling and beam quality improvement are feasible by applying a double-crystal orthogonal thermal compensation scheme to distribute the thermal load ${ }^{[47]}$. Because the beam size needs adapting to avoid damage, the lack of an appropriate optical imaging system for the pump prevented us from implementing further amplification based on the double-crystal geometry. Work based on this scheme is underway.

\section{Acknowledgements}

This work was supported by the Ministry of Science and Technology of the People's Republic of China (MOST) (No. 2017YFB0405202).

\section{References}

1. B. Luther-Davies, V. Z. Kolev, M. J. Lederer, N. R. Madsen, A. V. Rode, J. Giesekus, K. M. Du, and M. Duering, Appl. Phys. A 79, 1051 (2004).

2. M. Mero, Z. Heiner, V. Petrov, H. Rottke, F. Branchi, G. M. Thomas, and M. J. J. Vrakking, Opt. Lett. 43, 5246 (2018).

3. H. Fattahi, A. Schwarz, X. T. Geng, S. Keiber, D. E. Kim, F. Krausz, and N. Karpowicz, Opt. Express 22, 31440 (2014).

4. J. Ahrens, O. Prochnow, T. Binhammer, T. Lang, B. Schulz, M. Frede, and U. Morgner, Opt. Express 24, 8074 (2016).

5. R. Danilevicius, A. Zaukevicius, R. Budriunas, A. Michailovas, and N. Rusteika, Opt. Express 24, 17532 (2016).

6. M. Luhrmann, C. Theobald, R. Wallenstein, and J. A. L'Huillier, Opt. Express 17, 22761 (2009).

7. L. von Grafenstein, M. Bock, D. Ueberschaer, U. Griebner, and T. Elsaesser, Opt. Lett. 41, 4668 (2016).

8. M. Ueffing, R. Lange, T. Pleyer, V. Pervak, T. Metzger, D. Sutter, Z. Major, T. Nubbemeyer, and F. Krausz, Opt. Lett. 41, 3840 (2016)

9. H. Fattahi, A. Alismail, H. Wang, J. Brons, O. Pronin, T. Buberl, L. Vamos, G. Arisholm, A. M. Azzeer, and F. Krausz, Opt. Lett. 41, 1126 (2016).

10. T. Nubbemeyer, M. Kaumanns, M. Ueffing, M. Gorjan, A. Alismail, H. Fattahi, J. Brons, O. Pronin, H. G. Barros, Z.
Major, T. Metzger, D. Sutter, and F. Krausz, Opt. Lett. 42, 1381 (2017).

11. F. X. Morrissey, T. Y. Fan, D. E. Miller, and D. Rand, Opt. Lett. 42, 707 (2017)

12. J. Pouysegur, M. Delaigue, Y. Zaouter, C. Honninger, E. Mottay, A. Jaffres, P. Loiseau, B. Viana, P. Georges, and F. Druon, Opt. Lett. 38, 5180 (2013).

13. E. Caracciolo, M. Kemnitzer, A. Guandalini, F. Pirzio, A. Agnesi, and J. A. der Au, Opt. Express 22, 19912 (2014).

14. Z. Bai, Z. Fan, Z. Bai, F. Lian, Z. Kang, and W. Lin, Appl. Sci. 5, 359 (2015).

15. E. Caracciolo, A. Guandalini, F. Pirzio, M. Kemnitzer, F. Kienle, A. Agnesi, and J. A. der Au, Proc. SPIE 10082, 100821E (2017).

16. J. Dorring, A. Killi, U. Morgner, A. Lang, M. Lederer, and D. Kopf, Opt. Express 12, 1759 (2004).

17. P. Kroetz, A. Ruehl, A. L. Calendron, G. Chatterjee, H. Cankaya, K. Murari, F. X. Kartner, I. Hartl, and R. J. D. Miller, Appl. Phys. B 123, 126 (2017).

18. L. von Grafenstein, M. Bock, and U. Griebner, IEEE J. Sel. Top. Quantum Electron. 24, 3000213 (2018).

19. M. Grishin, V. Gulbinas, and A. Michailovas, Opt. Express 17, 15700 (2009).

20. M. Grishin, V. Gulbinas, and A. Michailovas, Opt. Express 15, 9434 (2007).

21. P. Gao, H. Lin, J. Li, J. Guo, H. Yu, H. Zhang, and X. Liang, Opt. Express 24, 13963 (2016).

22. P. Kroetz, A. Ruehl, G. Chatterjee, A. L. Calendron, K. Murari, H. Cankaya, P. Li, F. X. Kartner, I. Hartl, and R. J. D. Miller, Opt. Lett. 40, 5427 (2015).

23. L. von Grafenstein, M. Bock, G. Steinmeyer, U. Griebner, and T. Elsaesser, Laser Photon. Rev. 10, 123 (2016).

24. K. Mecseki, D. Bigourd, S. Patankar, N. H. Stuart, and R. A. Smith, Appl. Opt. 53, 2229 (2014).

25. A. V. Okishev, C. Dorrer, V. I. Smirnov, L. B. Glebov, and J. D. Zuegel, Opt. Express 15, 8197 (2007).

26. H. Lin, J. Li, J. He, and X. Liang, Chin. Opt. Lett. 9, 081404 (2011).

27. A. F. Kornev, R. V. Balmashnov, I. G. Kuchma, A. S. Davtian, and D. O. Oborotov, Opt. Lett. 43, 4394 (2018).

28. M. Long, L. Chen, M. Chen, and G. Li, Appl. Phys. B 122, 142 (2016).

29. D. W. E. Noom, S. Witte, J. Morgenweg, R. K. Altmann, and K. S. E. Eikema, Opt. Lett. 38, 3021 (2013).

30. Z. Bai, Z. Bai, C. Yang, L. Chen, M. Chen, and G. Li, Opt. Laser Technol. 46, 25 (2013).

31. Z. Peng, M. Chen, C. Yang, L. Chang, and G. Li, Jpn. J. Appl. Phys. 54, 028001 (2015).

32. D. A. Clubley, A. S. Bell, and G. Friel, Proc. SPIE 6871, 68711D (2008). 
33. F. Harth, T. Ulm, M. Luhrmann, R. Knappe, A. Klehr, T. Hoffmann, G. Erbert, and J. A. L'Huillier, Opt. Express 20, 7002 (2012).

34. M. Chen, L. Chang, C. Yang, L. Chen, and G. Li, Chin. J. Lasers 40, 0602010 (2013).

35. B. Zhang, G. Li, M. Chen, Z. Zhang, and Y. Wang, Opt. Lett. 28, 1829 (2003).

36. A. I. Vodchits, V. A. Orlovich, and P. A. Apanasevich, J. Appl. Spectrosc. 78, 918 (2012).

37. W. Koechner, Solid State Laser Engineering, 6th edition (Springer, New York, 2006).

38. H. Yu, J. Liu, H. Zhang, A. A. Kaminskii, Z. Wang, and J. Wang, Laser Photon. Rev. 8, 847 (2014).

39. R. Lavi and S. Jackel, Appl. Opt. 39, 3093 (2000).

40. R. Lavi, S. Jackel, Y. Tzuk, M. Winik, E. Lebiush, M. Katz, and I. Paiss, Appl. Opt. 38, 7382 (1999).
41. T. Jensen, V. G. Ostroumov, J. P. Meyn, G. Huber, A. I. Zagumennyi, and I. A. Shcherbakov, Appl. Phys. B 58, 373 (1994).

42. L. McDonagh, R. Wallenstein, R. Knappe, and A. Nebel, Opt. Lett. 31, 3297 (2006).

43. J. Kleinbauer, R. Knappe, and R. Wallenstein, Appl. Phys. B 81, 163 (2005).

44. M. Grishin and A. Michailovas, Advances in Solid State Lasers: Development and Applications (Intech, Vukovar, 2010).

45. K. T. Alligood, T. D. Sauer, and J. A. Yorke, Chaos: An Introduction to Dynamical Systems (Springer, New York, 1996).

46. H. Lin, J. Guo, P. Gao, H. Yu, and X. Liang, Opt. Express 24, 13957 (2016).

47. D. Sun, H. Lin, J. Guo, W. Wang, and X. Liang, Opt. Lett. 43, 4346 (2018). 